\title{
Surface morphology, structural and electrical properties of RF-sputtered ITO thin films on Si substrates
}

\author{
A LEBBAD ${ }^{1}$, L KERKACHE ${ }^{1, *}$ (D, A LAYADI ${ }^{1}$, F LEROY $^{2}$, B ALSHEHRI ${ }^{2}$ and E DOGHECHE ${ }^{2}$ \\ ${ }^{1}$ L.E.S.I.M.S, Département de Physique, Université Ferhat Abbas, Sétif 1, 19000 Sétif, Algeria \\ ${ }^{2}$ Institut d'Electronique Microélectronique et Nanotechnologie IEMN - UMR 8520 CNRS, Université Valenciennes et du \\ Hainaut Cambrésis, Le Mont Houy, 59309 Valenciennes Cedex, France \\ *Author for correspondence (kerkachelaid@yahoo.fr, laid.kerkache@univ-setif.dz)
}

MS received 2 May 2017; accepted 13 October 2017; published online 19 May 2018

\begin{abstract}
Series of indium tin oxide (ITO) thin films were deposited onto Si(100) substrate by RF sputtering. The film thickness ranges from 61 to $768 \mathrm{~nm}$. X-ray diffraction (XRD), scanning electron microscopy (SEM) and atomic force microscopy (AFM) experiments were performed to study the structure and the surface morphology of these films. The electrical properties were obtained by a Hall effect measurement system; the electrical resistivity $\rho$, the carrier concentration $n$ and the mobility $\mu$ were measured. Annealing experiments were carried out in the air at $400^{\circ} \mathrm{C}$ for 60 min. The different physical parameters were investigated as a function of thickness before and after annealing. The effects of power and deposition rate were also addressed. We noted that the behaviour of some parameters with thickness is different before and after annealing. All these results are discussed and correlated in this article. Also, the results of the present ITO/Si system were compared to those of the ITO/glass, we have previously published.
\end{abstract}

Keywords. ITO thin films; sputtering; structure; electrical properties; AFM; Hall effect.

\section{Introduction}

Indium tin oxide (ITO) is one of the most used oxides because of its excellent properties such as a good transparency in the visible, high infrared reflectivity and UV absorption, good electric conductivity, mechanical resistance and adherence with the substrate. These interesting properties make ITO a material of choice for extensive technological applications, especially in solar cells [1-3], flat panels [4], gas sensor [5], light-emitting diodes [6] and conductive electrode [7]. Various techniques are used to prepare the ITO films such as radio frequency (RF) sputtering [8,9], DC sputtering [10], RF/DC magnetron sputtering [11,12], RF reactive sputtering [13], e-beam evaporation [14], pulsed laser deposition (PLD) [15], spray pyrolysis [16], ion beam sputter deposition [17] and sol-gel [18]. The choice of the technique and the preparation conditions are the critical points of a research topic since the structural, optical and electrical properties of these films are strongly associated with the elaboration process and operating conditions [19].

In this work, we present experimental results pertaining to the structural and electrical properties of RF-sputtered ITO thin films deposited onto $\mathrm{Si}(100)$. Also the effect of annealing at $T=400^{\circ} \mathrm{C}$ during $60 \mathrm{~min}$ will be discussed.

The purposes of the present work are (i) to investigate the electrical properties of RF-sputtered ITO/Si in a given thickness range (61-768 nm) and to see if this system present properties that are suitable for some of the applications cited above, (ii) to find out how annealing at $400^{\circ} \mathrm{C}$ would modify these transport properties, (iii) to correlate between the observed electrical properties in one hand, the structural ones and some parameters (thickness and power) on the other; this may give insight on the different origins for the electrical behaviour of such a system, (iv) to investigate the effect of substrate $\mathrm{Si}$ on the structural and electrical properties; substrates were found to affect differently the ITO physical properties and (v) to compare the behaviour of such a system with that of other ones such as the RF-sputtered ITO/glass thin films that we have previously published [20].

Moreover, recently, interest arose on ferroelectric thin films such as the lead zirconate titanate, $\mathrm{PbZr} 0.53 \mathrm{TiO}_{3} 0.47$ (commonly called PZT) because of their many potential applications in electronic devices. The PZT/ITO (i.e., the use of ITO as a bottom electrode) and the ITO/PZT/ITO structures (where ITO is used for both the electrodes) have attracted a lot attention [21]. These structures are generally deposited on Si substrate [22]. The present study of ITO/Si may thus be of interest for researchers in this field.

\section{Experimental}

ITO thin films were deposited at room temperature (RT) onto silicon substrate with (100) orientation by RF sputtering system with $99.99 \%$ tin-doped indium oxide $\left(90 \mathrm{wt} \% \mathrm{In}_{2} \mathrm{O}_{3}\right.$ and $10 \mathrm{wt} \% \mathrm{SnO}_{2}$ ) target in an argon atmosphere. The vacuum chamber was evacuated down to a pressure of $10^{-6}$ mbar. 
Table 1. The deposition conditions of the RF-sputtered ITO/Si thin films (power (W), deposition time (min), thickness $(\mathrm{nm})$, deposition rate $\left.\left(\mathrm{nm} \mathrm{min}{ }^{-1}\right)\right)$ along with the internal strain $\varepsilon(\%)$ and the surface roughness (rms).

\begin{tabular}{|c|c|c|c|c|c|c|c|}
\hline \multirow[b]{2}{*}{ Power (W) } & \multirow{2}{*}{$\begin{array}{l}\text { Deposition } \\
\text { time (min) }\end{array}$} & \multirow{2}{*}{$\begin{array}{l}\text { Thickness } \\
(\mathrm{nm})\end{array}$} & \multirow{2}{*}{$\begin{array}{l}\text { Deposition rate } \\
\left(\mathrm{nm} \mathrm{min} \min ^{-1}\right)\end{array}$} & \multicolumn{2}{|c|}{ Strain $\varepsilon(\%)$} & \multicolumn{2}{|c|}{ Roughness rms (nm) } \\
\hline & & & & RT & $T=400^{\circ} \mathrm{C}$ & RT & $T=400^{\circ} \mathrm{C}$ \\
\hline 75 & 10 & 61 & 6.1 & -0.87 & -0.67 & 5.6 & 4.98 \\
\hline 100 & 10 & 128 & 12.8 & -0.67 & -0.37 & 0.82 & 1.36 \\
\hline 125 & 10 & 182 & 18.2 & -0.77 & -0.37 & 1.62 & 1.92 \\
\hline 125 & 15 & 264 & 17.7 & -0.77 & -0.47 & 2.40 & 2.66 \\
\hline 125 & 45 & 768 & 17.1 & -0.47 & -0.28 & 2.89 & 4.32 \\
\hline
\end{tabular}

Then argon gas was introduced into the chamber up to the required pressure of about $0.6 \mathrm{~Pa}\left(6 \times 10^{-3} \mathrm{mbar}\right)$. The thicknesses ' $t$ ' of these samples are between 61 and $768 \mathrm{~nm}$. The Si substrates were subjected to a chemical cleaning. Then, they were immersed successively in a bath of dichloromethane, acetone and isopropyl, and they were dried by argon gas.

The deposition conditions are summarized in table 1 . Three samples were made at the same deposition time $(10 \mathrm{~min})$ and with three different powers $\mathrm{P}(75,100$ and $125 \mathrm{~W})$. We see that there is a linear increase of the deposition rate $v$ with increasing $P$ : from $6.1 \mathrm{~nm} \mathrm{~min}-1$ at $75 \mathrm{~W}$ to $12.8 \mathrm{~nm} \mathrm{~min}^{-1}$ at $100 \mathrm{~W}$ and to $18.2 \mathrm{~nm} \mathrm{~min}^{-1}$ at $125 \mathrm{~W}$, i.e., there is an increase of $0.24 \mathrm{~nm} \mathrm{~min}^{-1}$ per Watt. The last three samples of table 1 were elaborated at the same power $(125 \mathrm{~W})$ and different deposition times (10, 15 and $45 \mathrm{~min})$. A fixed power led to a fixed deposition rate of $17.6( \pm 0.5) \mathrm{nm} \mathrm{min}^{-1}$ for the three films.

The structural properties of ITO thin films were carried out by an X-ray diffractometer (Philips, X'Pert PRO Console, in the $2 \theta$ mode) with $\mathrm{CuK} \alpha$ radiation at a wavelength $\lambda$ equal to $1.54056 \AA$. The surface morphology and topography were determined by means of the scanning electron microscopy (SEM) and the atomic force microscope (AFM). The electrical properties were studied by a Hall effect measurement system (HMS-5300 Ver 5.8.0.7, ECOPIA) where $B=0.56 \mathrm{~T}$, $I=1 \mathrm{~mA}$ and $T=300 \mathrm{~K}$. Our samples were annealed in air, at $T=400^{\circ} \mathrm{C}$ during $60 \mathrm{~min}$; after annealing, we have characterized these films by the same techniques (XRD, SEM, AFM and Hall effect measurement).

\section{Results and discussion}

\subsection{Structural properties}

For the as-deposited thin films, the XRD of the ITO films (figure 1a) shows that all samples are polycrystalline with different diffraction planes, such as (222), (400) and (521). As the thickness increases, new diffraction peaks appear like (310) and (321). We notice that no characteristic peaks of Sn, $\mathrm{SnO}$ or $\mathrm{SnO}_{2}$ appeared, this means that the tin have replaced the indium in the cubic lattice in substitution positions [2,9].
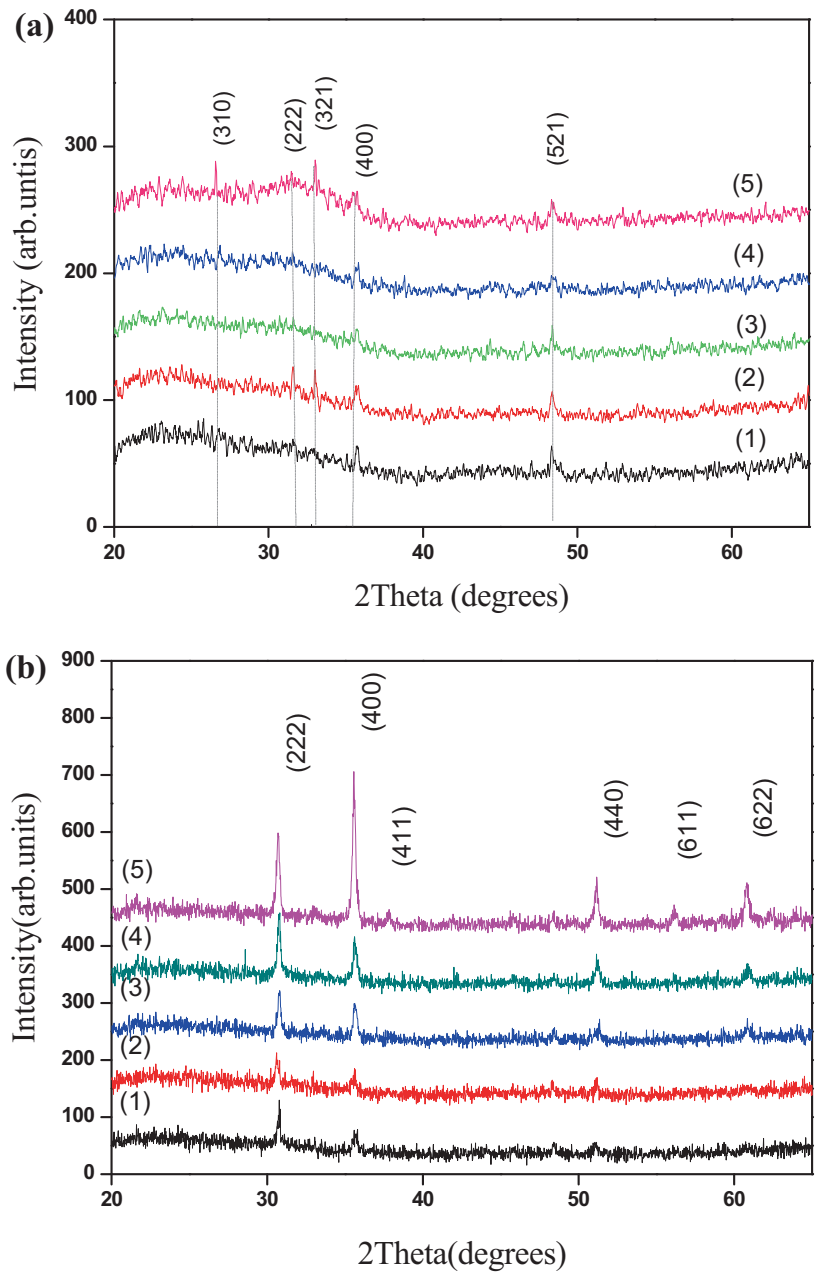

Figure 1. X-ray spectra of ITO thin films for different thicknesses: (a) as-deposited and (b) annealed samples $\left(T=400^{\circ} \mathrm{C}\right.$ for $\left.1 \mathrm{~h}\right)$; with (1) 61, (2) 128, , (3) 182, (4) 264 and (5) $768 \mathrm{~nm}$.

From the computation of the relative intensity of the peaks, we noticed that most of our samples have a $\langle 100\rangle$ texture. It is interesting to note that in a previous work [20] on RFsputtered ITO onto glass substrate; we observed a change in the texture with thickness, i.e., the texture changes from $\langle 100\rangle$ to $\langle 111\rangle$ for $t>582 \mathrm{~nm}$. For ITO/Si, the texture is $\langle 100\rangle$ over 
all the thickness range even for the thicker film $(768 \mathrm{~nm})$; this may be attributed to the crystallographic structure of the substrate: Si with $\langle 100\rangle$ orientation in the present work and amorphous glass in the previous one.

After annealing, we observed a strong improvement of the structural properties where the peaks become more intense (compare figure $1 \mathrm{a}$ and $\mathrm{b}$ ). Annealing did not change the preferential orientation. In fact, the $\langle 100\rangle$ preferential direction becomes stronger. Once again there are no peaks corresponding to $\mathrm{Sn}, \mathrm{SnO}$ or $\mathrm{SnO}_{2}$, or to a new phase (such as a possible phase at the interface); thus, annealing did not change the atomic arrangement and did not induce interdiffusion at the interface which could lead to a formation of an alloy.

We deduce the lattice parameter from the XRD spectra using the Bragg equation, and the relation linking the interplanar spacing to Miller indices $(h k l)$ which is given in a cubic system by the following equation:

$$
a=\frac{\lambda}{2 \sin \theta} \cdot \sqrt{h^{2}+k^{2}+l^{2}}
$$

where $(h, k, l)$ are the Miller indices, $\theta$ the incidence angle and $\lambda$ the X-ray photon wavelength. The values of the lattice parameter for the most intense peak are shown in figure 2 . We note that the lattice parameter for the (400) plane, which corresponds to the preferred direction is lower than that of the powder for all the films (the lattice constant of the $\mathrm{In}_{2} \mathrm{O}_{3}$ powder is equal to $10.118 \AA$ [23]), and in general, the lattice parameter increases with the thickness. We therefore, conclude that our films are subject to a compressive stress formed during deposition; the lattice parameter becomes closer to the bulk one for higher thicknesses (see figure 2). A similar result was reported by Manavizadeh et al [2], where the lattice parameter of ITO films prepared by RF sputtering on silicone substrates of orientation (100) and type-p (100), is lower than that of the powder.

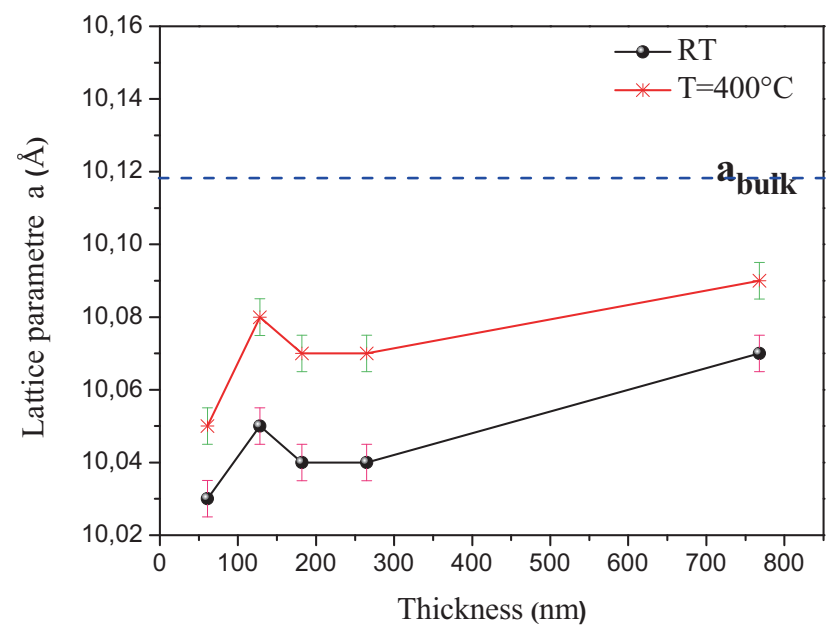

Figure 2. The variation of the lattice parameter as a function of thickness for ITO/Si thin films before and after annealing at $T=$ $400^{\circ} \mathrm{C}$ for $1 \mathrm{~h}$.
We note that the lattice parameter increases after annealing and approaches the powder value for most samples (figure 2). This indicates a reduction in the compressive stress; i.e., annealing relieved the internal stress in the films.

Another parameter used to study the film's internal stress is the out-of-plane strain $\varepsilon$ defined as $\varepsilon=a-a_{\text {bulk }} / a_{\text {bulk }}$, where $a$ and $a_{\text {bulk }}$ denote the measured and the bulk lattice parameter values. Values of $\varepsilon$ are shown in table 1 . The all $\varepsilon$ values are negative and small (in absolute values), $<1 \%$ for all the samples. Thus, the compressive stress is small in our samples and decreases even further with film thickness and annealing.

The XRD analysis also allows us to calculate the grain size, which may have an important role in the study of physical properties of materials as mentioned in the sub-section 'electrical properties'. The average grain size is determined from the Scherrer equation [24]:

$$
D=\frac{0.9 \lambda}{\Delta 2 \theta \cdot \cos \theta}
$$

$\lambda$ is the wavelength of the $\mathrm{X}$-ray radiation (1.54056 $\AA$ ), $\theta$ the diffraction angle of $\mathrm{X}$-ray and $\Delta 2 \theta$ the width at half height of the corresponding peaks expressed in radians. We calculated the grain size for the preferential orientation (400). The variation of $D$ with film thickness is shown in figure 3 . For as-deposited films, we observe an overall decrease of $D$ with $t$; in fact, besides the first large decrease between $t=61 \mathrm{~nm}$ ( $D=42.5 \mathrm{~nm})$ and $t=128 \mathrm{~nm}(D=16.7 \mathrm{~nm})$, the $D$ values seem to stabilize as $t$ is increased further. Annealing led to an increase in $D$ for most samples.

Also, for annealed samples, $D$ increases in a linear fashion from 26.8 (at $t=61 \mathrm{~nm}$ ) to $53.6 \mathrm{~nm}(t=264 \mathrm{~nm})$, then decreases for the thickest film.

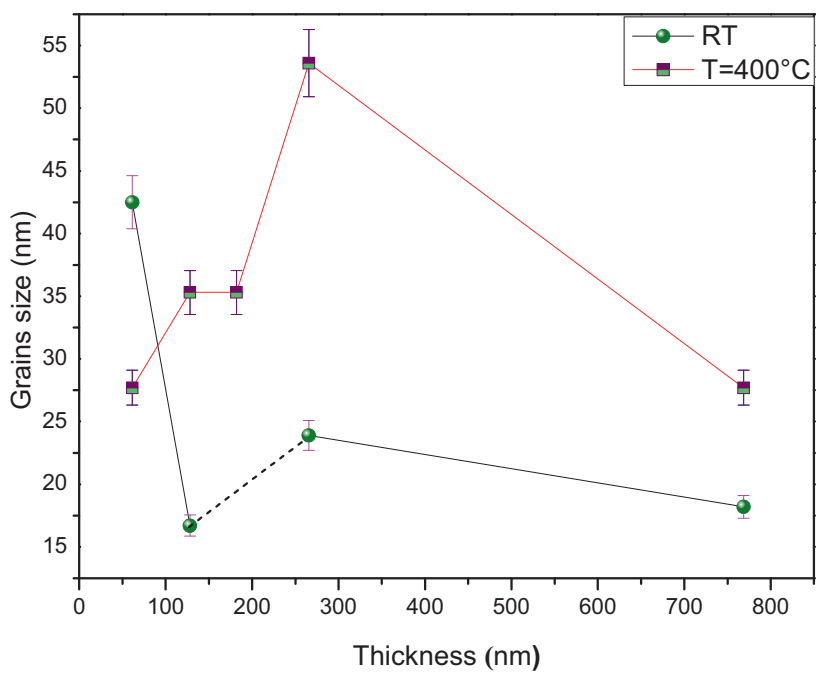

Figure 3. The variation of grain size as a function of thickness for ITO/Si thin films before and after annealing at $T=400^{\circ} \mathrm{C}$ for $1 \mathrm{~h}$. 


\subsection{Surface morphology}

The morphology of the as-deposited and annealed (at $T=$ $400^{\circ} \mathrm{C}$ for $1 \mathrm{~h}$ ) ITO thin films for various thicknesses is illustrated in figures 4 and 5. Examples of SEM surface images are shown along with the cross-sectional SEM micrograph for the
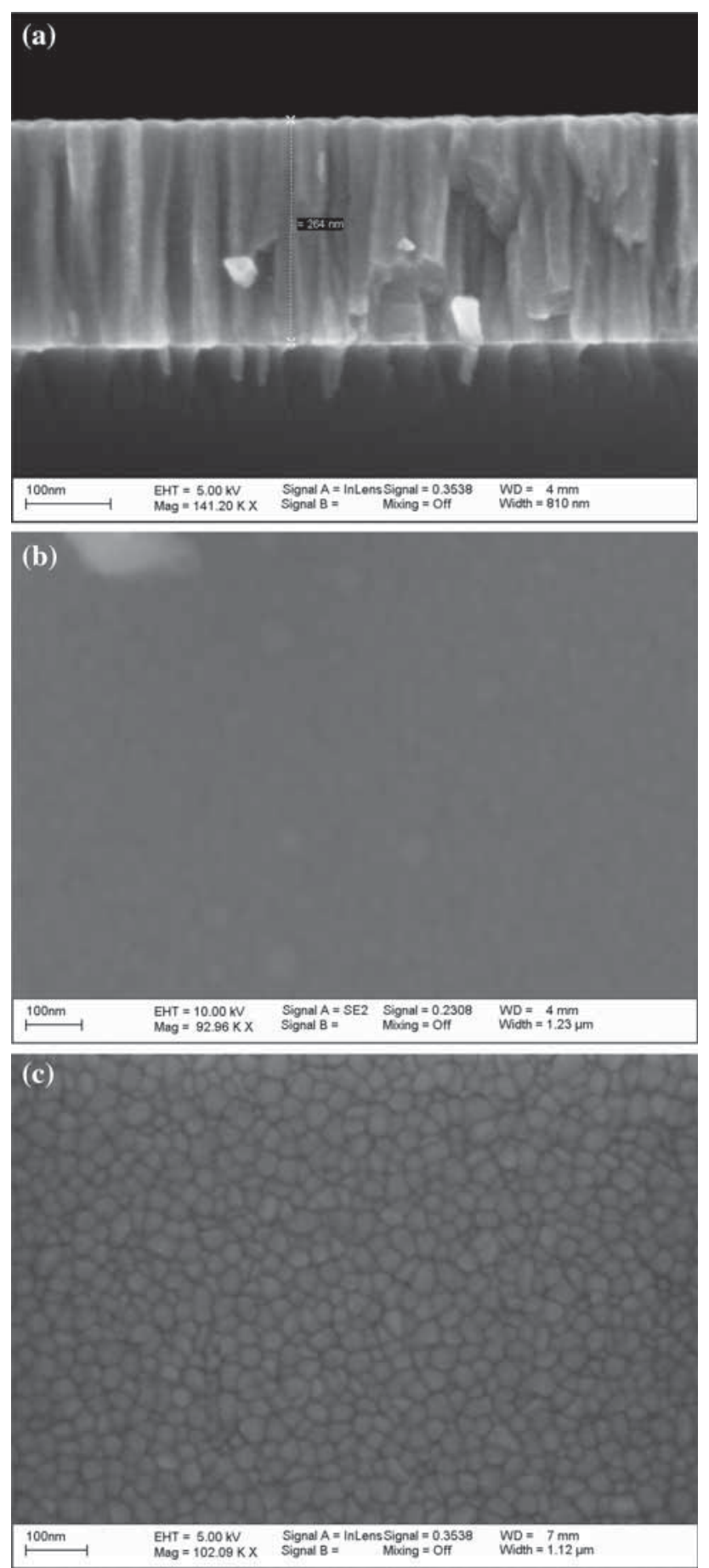

Figure 4. SEM images of $264 \mathrm{~nm}$ thick ITO/Si sample: (a) crosssectional SEM micrograph, (b) before annealing and (c) after annealing.
$264 \mathrm{~nm}$ thick sample; the micrograph of the cross-section of the $264 \mathrm{~nm}$ thick is shown. Grains with columnar structure are visible in the image (figure $4 \mathrm{a}$ ). The study of the morphology by SEM confirms the polycrystalline nature of ITO films and shows that the structure is smooth with the existence of small grains (figure 4b). After annealing, the SEM image shows that the structure is also smooth with larger grains (figure $4 \mathrm{c}$ ), confirming the behaviour of the $D$ values inferred from the XRD.

In figure 6, we show the AFM images (2D and 3D) of the surface morphologies for as-deposited (figure 6a) and annealed (figure 6b) ITO thin films with different thicknesses. The AFM analysis provides information on the morphology of ITO films, where the surface appears smooth with the existence of pyramid-shaped grains. Values of the surface roughness, the root mean square (rms) of ITO films were derived and are shown in table 1 . Besides the thinner sample $(t=$ $61 \mathrm{~nm})$, which is characterized by a relatively high rms value $(5.6 \mathrm{~nm})$, we note a monotonous increase of the roughness from 0.82 to $2.89 \mathrm{~nm}$ when the thickness is increased from 128 to $768 \mathrm{~nm}$. The rms value of the $128 \mathrm{~nm}$ thick sample (with the lowest roughness equal to $0.82 \mathrm{~nm}$ ) is comparable to that $(0.71 \mathrm{~nm})$ obtained by DC magnetron sputtering reported by Song et al [12]. However, our value $(0.82 \mathrm{~nm})$ is very small compared to that reported by Kim and Gulmor [25], which is equal to $3.90 \mathrm{~nm}$ in their ITO/glass films prepared by RF sputtering. Also, for RF-sputtered ITO/glass [20], the rms value was found to be $3.90 \mathrm{~nm}$ for $t=699 \mathrm{~nm}$ to be compared with $2.90 \mathrm{~nm}$ measured in the present work for a $768 \mathrm{~nm}$ thick ITO/Si sample; thus, the surface roughness is smaller for a Si substrate than for a glass one. Hence, in both our previous works [20] and that of Kim et al [25], the surface roughness in RF-sputtered ITO/glass is found larger than the one seen in the present ITO/Si; we believe that this fact may be due to the different growth modes in glass and Si substrates.

The root-mean-square (rms) of these films increases after annealing; while for the as-deposited films, the rms values range from 0.82 to $2.89 \mathrm{~nm}$ (excluding the thinnest sample), after annealing, the values are between 1.36 and $4.32 \mathrm{~nm}$ (see table 1).

It is worth noting the behaviour of thinnest film $(61 \mathrm{~nm})$, which seems to be different from that of the other samples. It is characterized by a very high rms value $(5.6 \mathrm{~nm})$ compared to other samples where the rms values monotonously increases from 0.82 (for $t=128 \mathrm{~nm}$ ) to $2.89 \mathrm{~nm}(t=768 \mathrm{~nm}$ ). Also, the $61 \mathrm{~nm}$ thick sample has a quite high $D$ value $(42.5 \mathrm{~nm})$ compared to other samples, where $D$ is between 16.7 and $23.9 \mathrm{~nm}$. Remember that this sample was made at the lowest power $(75 \mathrm{~W})$ and the lowest deposition rate $\left(6.1 \mathrm{~nm} \mathrm{~min}^{-1}\right)$. Thus, we believe that this departure from the trend of other samples is probably not due to the thickness, but to the power and the deposition rate. On the other hand, even though the $128 \mathrm{~nm}$ thick sample was made at a power $100 \mathrm{~W}$, less than the other three samples $(125 \mathrm{~W})$, its behaviour fits well in the trend of other samples, i.e., no effect of power is seen between samples made at 125 and $100 \mathrm{~W}$, contrary to the one made at 75 W. 

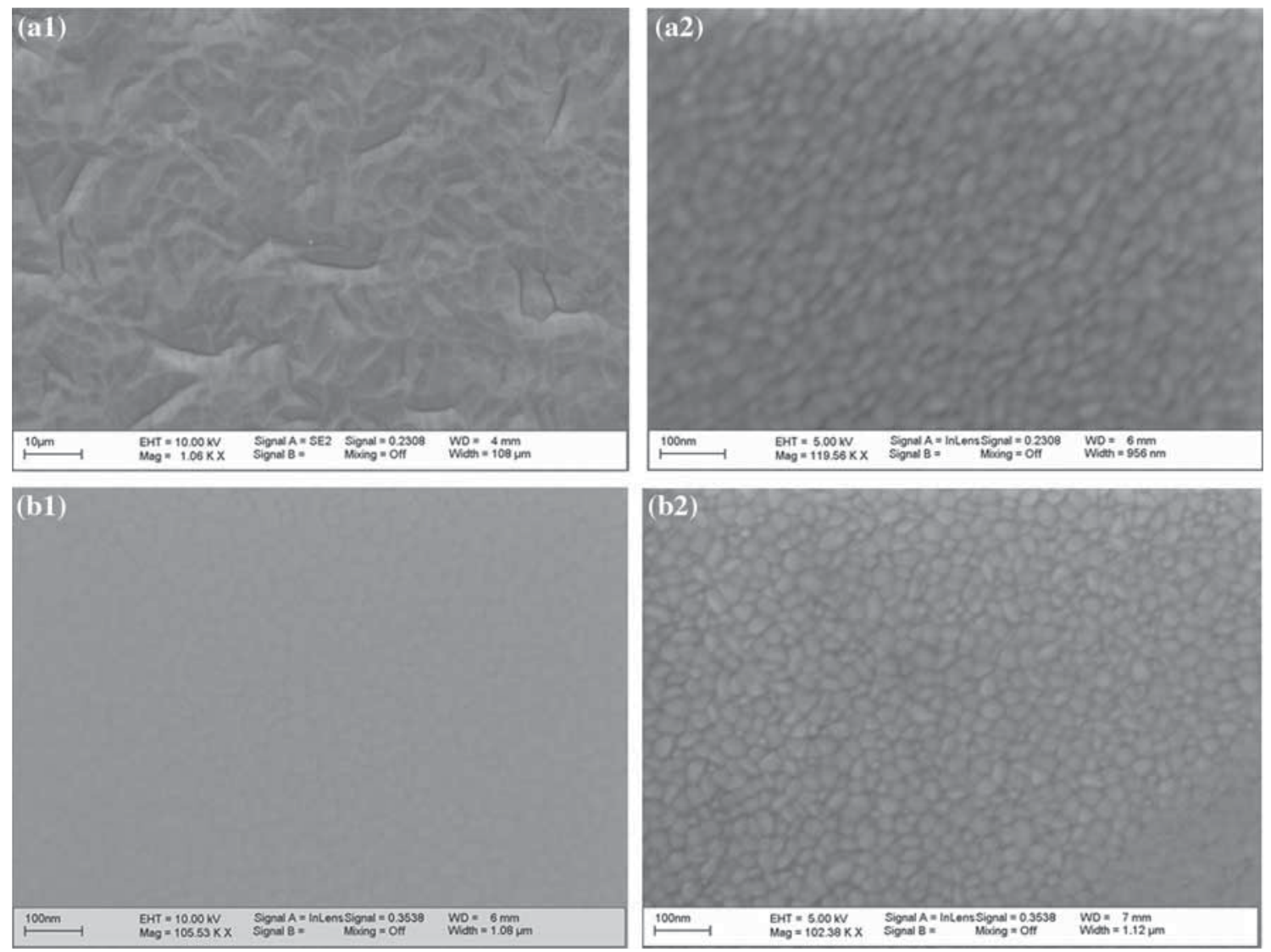

Figure 5. SEM images of (a) 182 and (b) $768 \mathrm{~nm}$ thick ITO/Si samples: (1) before and (2) after annealing.

\subsection{Electrical properties}

In table 2, we show the electrical properties of ITO thin films measured by Hall effect measurement system for as-deposited and annealed samples. Also, the electrical resistivity $\rho$ is plotted against the film thickness in figure 7 .

For the as-deposited samples, we note that $\rho$ increases with increasing thickness (see figure 7). This trend is opposite to that one would expect for thin films because of the scattering at the surface. Thus, the scattering at the surface is not the most important factor for the $\rho$ values in this thickness range. On the other hand, we see a correlation between $\rho$ and the grain size $D$. Indeed, as $D$ decreases, $\rho$ increases for most samples. This indicates that scattering at the grain boundaries is the major origin of the $\rho$ behaviour. Note however, that for the $264 \mathrm{~nm}$ thick sample, we observe an increase of $D$ (compared to the $128 \mathrm{~nm}$ thick sample) and also an increase in the electrical resistivity; for this particular case, the grain boundary scattering cannot explain this behaviour. Other mechanisms such as defects or impurities might be responsible for the increase of $\rho$ in this sample.

After annealing, we see a large decrease of $\rho$ (see figure 7), down to a value which amounts to about $5.5 \times 10^{-4} \Omega$.cm; we see a small increase of $\rho$ from 5.52 to $6.93 \times 10^{-4} \Omega . \mathrm{cm}$ as $t$ increases from 61 to $264 \mathrm{~nm}$, however, we believe that within the measurement uncertainties, the $\rho$ value seems to be independent of thickness. Once again, annealing led to an increase in $D$ and a decrease of $\rho$ confirming that the grain boundary scattering affects the $\rho$ values. Note, once again that while scattering at the grain boundaries might explain the variation of the electrical resistivity $\rho$ before and after annealing, it is not sufficient to explain the electrical behaviour with thickness, other mechanisms should also be considered such as defects and impurities. Also, the stronger texture after annealing might have contributed to the decrease of $\rho$ as was noted previously in RF-sputtered ITO/glass thin films [20]. For comparison, one cite the work by Manavizadeh et al [9] who showed that the resistivity decreases from $19 \times 10^{-4} \Omega$.cm to about $1 \times 10^{-4} \Omega$.cm after annealing in air at $T=400^{\circ} \mathrm{C}$ for ITO films deposited by RF sputtering on glass substrate. Song et al [12] have elaborated ITO/glass (thickness $200 \mathrm{~nm}$ ) by magnetron sputtering, they found that ITO films annealed at $600^{\circ} \mathrm{C}$ have a resistivity of $1.6 \times 10^{-4} \Omega$.cm. Also, Balasundaraprabhu et al [26] studied the effect of annealing in DC magnetron-sputtered ITO/Si thin films; they found out that annealing at a temperature up to $300^{\circ} \mathrm{C}$ does improve the properties of the film 
(a)
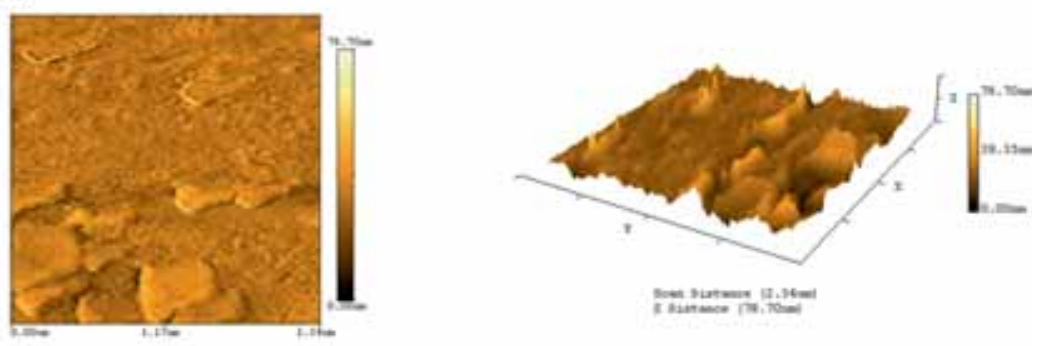

inington

(1)
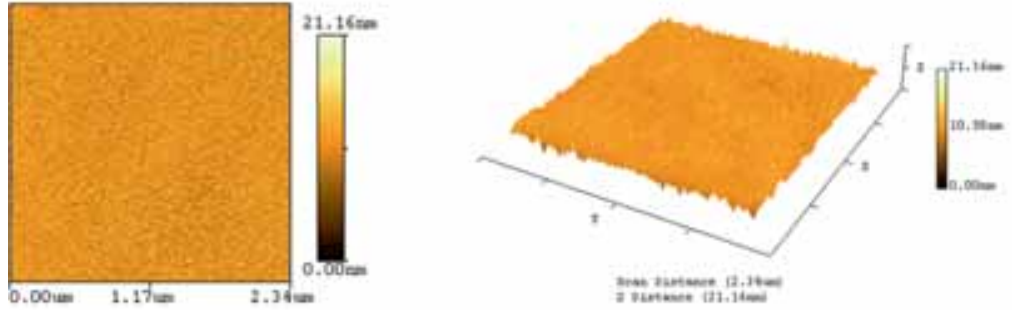

(2)
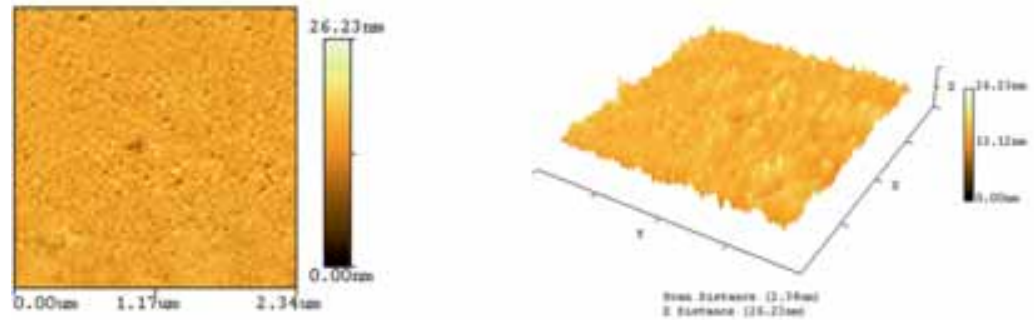

(3)
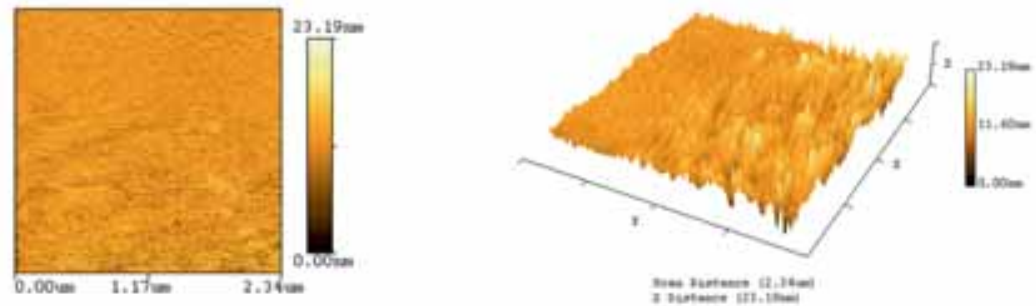

(4)
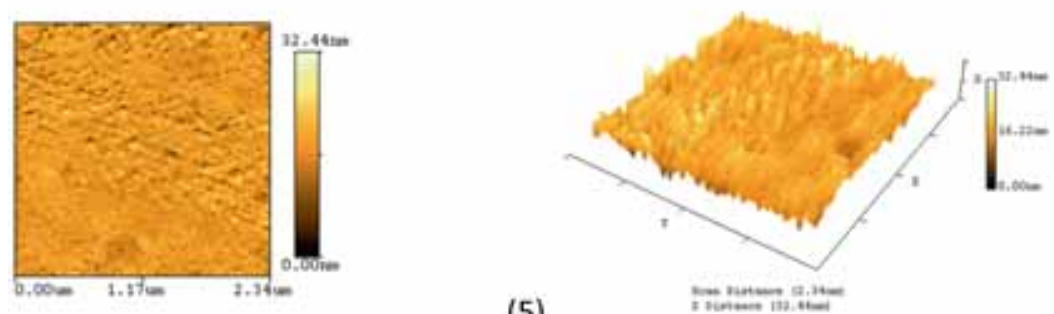

(5)

Figure 6. (a) AFM images (2D and 3D) of ITO thin films for different thicknesses: (1) 61, (2) 128, (3) 182, (4) 264 and (5) $768 \mathrm{~nm}$. (b) AFM images of ITO thin films after annealing at $T=400^{\circ} \mathrm{C}$ for $1 \mathrm{~h}$ for different thicknesses: (1) 61, (2) 128, (3) 182, (4) 264 and (5) $768 \mathrm{~nm}$. 
(b)
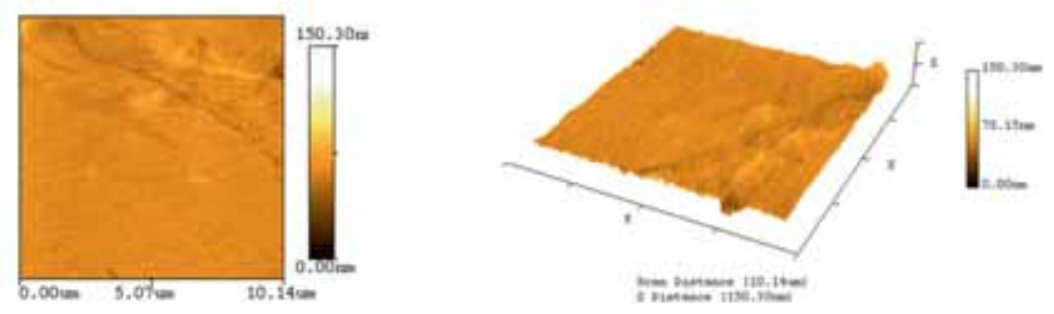

(1)
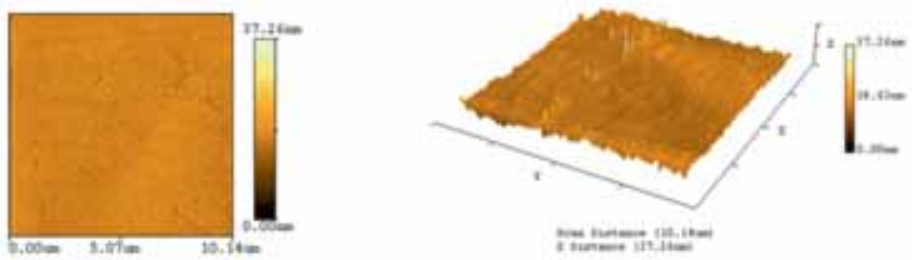

(2)
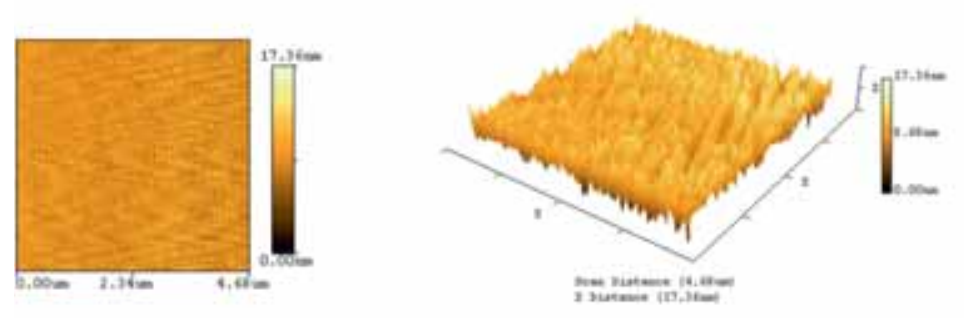

(3)
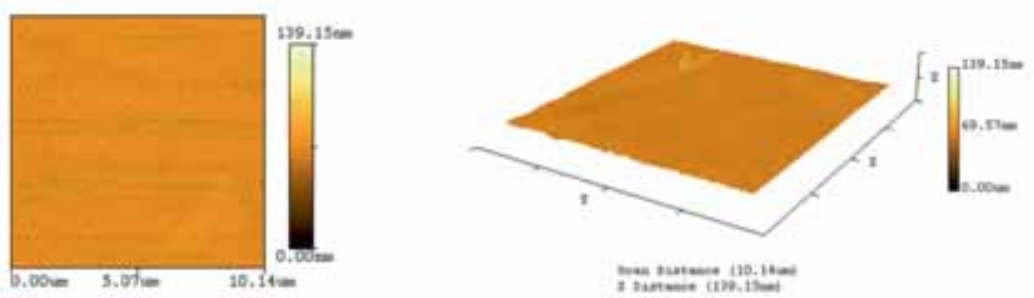

(4)
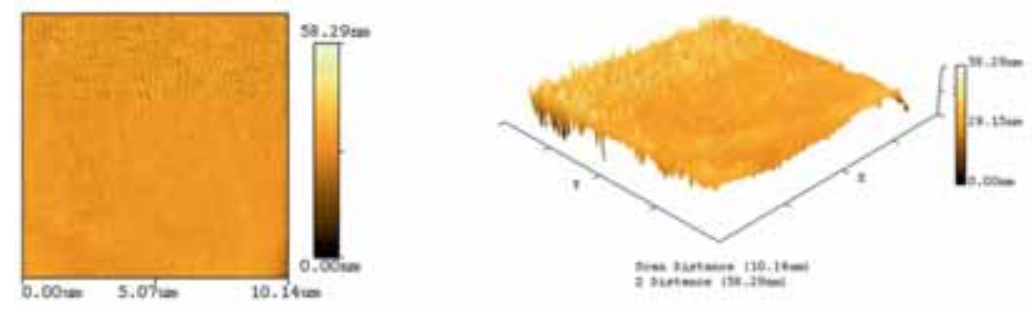

(5)

Figure 6. Continued. 
Table 2. Electrical properties: the bulk concentration $n$, the sheet concentration $n_{\mathrm{s}}$, the electrical resistivity $\rho$, the magnetoresistance and the mobility $\mu$ as a function of thickness $t$ for as-deposited ITO/Si thin films and annealed at $T=400^{\circ} \mathrm{C}$ for $1 \mathrm{~h}$.

\begin{tabular}{|c|c|c|c|c|c|c|c|c|c|c|}
\hline \multirow[b]{2}{*}{ Thickness (nm) } & \multicolumn{2}{|c|}{$\begin{array}{l}\text { Bulk concentration } \\
\times 10^{19}\left(/ \mathrm{cm}^{3}\right) \\
\end{array}$} & \multicolumn{2}{|c|}{$\begin{array}{l}\text { Sheet concentration } \\
\times 10^{15}\left(/ \mathrm{cm}^{2}\right) \\
\end{array}$} & \multicolumn{2}{|c|}{$\begin{array}{l}\text { Resistivity } \\
\times 10^{-4}\left(\Omega \mathrm{cm}^{-1}\right) \\
\end{array}$} & \multicolumn{2}{|c|}{$\begin{array}{l}\text { Magnetoresistance } \\
(\Omega)\end{array}$} & \multicolumn{2}{|c|}{$\begin{array}{c}\text { Mobility } \\
\left(\mathrm{cm}^{2} \mathrm{~V}^{-1}\right)\end{array}$} \\
\hline & RT & $T=400^{\circ} \mathrm{C}$ & RT & $T=400^{\circ} \mathrm{C}$ & $\mathrm{RT}$ & $T=400^{\circ} \mathrm{C}$ & RT & $T=400^{\circ} \mathrm{C}$ & RT & $T=400^{\circ} \mathrm{C}$ \\
\hline 61 & 18.11 & 36.94 & 1.11 & 2.27 & 37.54 & 5.52 & 0.37 & 0.15 & 9.18 & 30.72 \\
\hline 128 & 3.31 & 44.93 & 0.42 & 5.76 & 42.04 & 5.53 & 0.82 & 0.01 & 44.81 & 27.44 \\
\hline 182 & 1.56 & 69.28 & 0.28 & 12.61 & 58.29 & 5.61 & 1.23 & 0.03 & 68.56 & 16.07 \\
\hline 264 & 1.21 & 76.44 & 0.32 & 20.29 & 85.43 & 6.93 & 1.09 & 0.02 & 60.48 & 11.79 \\
\hline
\end{tabular}

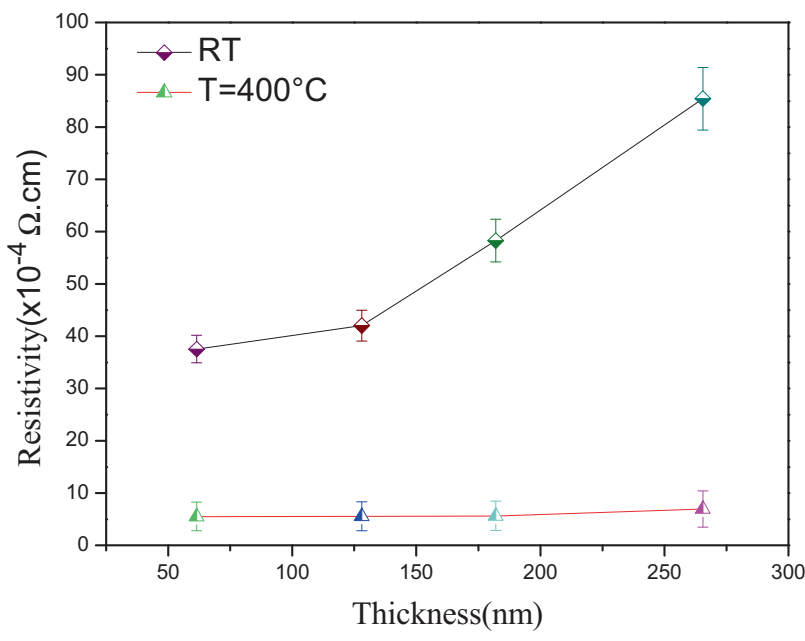

Figure 7. The electrical resistivity $v s$. thickness for the ITO/Si thin films before and after annealing at $T=400^{\circ} \mathrm{C}$ for $1 \mathrm{~h}$.

such as a reduction in the electrical resistivity $\rho$. However, they state that annealing at $400^{\circ} \mathrm{C}$ led to an increase of $\rho$ at about $7.5 \times 10^{-4} \Omega . \mathrm{cm}$ for a $300 \mathrm{~nm}$ thick sample, this increase is attributed by the authors to oxygen vacancy compensation [26]. Our value for comparable thickness (about $6.93 \times 10^{-4} \Omega . \mathrm{cm}$ ) in RF-sputtered ITO/Si films annealed under the same conditions $\left(400^{\circ} \mathrm{C}\right)$ is close to the one that they reported.

The magnetoresistance is the change $\Delta R$ of the electrical resistance upon the application of a magnetic field, i.e., $\Delta R=R(H)-R(0)$. The magnetoresistance is usually more important for ferromagnetic materials. Lately, work was done on ITO doped with ferromagnetic element [27]. We thought that measuring the magnetoresistance of pure ITO would be of interest for those working on doped-ITO, the values can be taken as references for comparison with ferromagnetic doped-ITO thin films. The values of $\Delta R$ are shown in table 2 . There is an overall increase in the magnetoresistance with $t$ for the as-deposited samples in the $0.37-1.23 \Omega$ range. This trend is identical to that of $\rho$ with $t$. After annealing, there is a decrease in the magnetoresistance for all samples (values range from 0.15 down to $0.01 \Omega)$.
The bulk carrier concentration $n$ was deduced from the Hall effect by measuring the Hall tension $V_{\mathrm{H}}$ given by [28]:

$$
V_{\mathrm{H}}=R_{\mathrm{H}} \frac{I}{t} B
$$

where $I$ is the current (equal to $1 \mathrm{~mA}$ in the present experiment), $t$ the film thickness and $B$ the magnetic induction applied normal to the film plane, $B=0.56 \mathrm{~T}$; the Hall constant $R_{\mathrm{H}}$ is given by

$$
R_{\mathrm{H}}=\frac{1}{n q},
$$

Here $q$ is the charge and $n$ the carrier concentration. The bulk carrier concentration $n$ decreases from 18.11 to $1.21 \times$ $10^{19} \mathrm{~cm}^{-3}$ as $t$ increases from 61 to $264 \mathrm{~nm}$ in the as-deposited samples (see table 2).

After annealing, we note an increase of $n$ for all thicknesses. Also, there is a monotonous increase of $n$ with $t$, from 36.94 to $76.44 \times 10^{19} \mathrm{~cm}^{-3}$, when $t$ increases from 61 to $264 \mathrm{~nm}$; this variation of $n$ with $t$ for the annealed samples is opposite to that of the as-deposited ones. We observe that the increase of $n$ after annealing is followed by a decrease of $\rho$. Thus, the variation of $n$ seems to be due to electrical resistivity as was pointed out in some studies, i.e., lower the resistivity, higher the concentration $n$. However, this relationship does not hold for the variation of $n$ and $\rho$ with $t$ for the annealed samples. Really, as $t$ increases from 61 to $264 \mathrm{~nm}$, we see a large increase of $n$ and also a small increase of $\rho$.

The sheet concentration $n_{\mathrm{s}}$ values, i.e., the concentration per unit area, are also shown in table 2. As a function of $t, n_{\mathrm{s}}$ decreases with $t$ before annealing and monotonously increases with $t$ after annealing. The concentration $n_{\mathrm{s}}$ has the same behaviour as the $n$ one. Annealing led to an increase of $n_{\mathrm{s}}$ for all samples. There is an overall decrease of $n_{\mathrm{s}}$ from 1.11 to $0.32 \times 10^{15} \mathrm{~cm}^{-3}$ for as-deposited and a monotonous increase from 2.27 to $20.29 \times 10^{15} \mathrm{~cm}^{-3}$ for the annealed ones when $t$ is increased from 61 to $264 \mathrm{~nm}$.

The carrier mobility $\mu$ is given by the following relation [29]:

$$
\mu=\frac{R_{\mathrm{H}}}{\rho} .
$$


The carrier mobility $\mu$ values were computed and are shown in table 2. As $t$ increases, there is an overall increases of $\mu$ with $t$ for as-deposited samples and a monotonous decrease (from 30.72 down to $11.79 \mathrm{~cm}^{2} \mathrm{Vs}^{-1}$ ) for the annealed ones. Annealing led to a decrease of $\mu$ for all the samples except the thinnest one made at the lowest power. Also, we note an inverse relationship between $n$ and $\mu$ in all cases. For asdeposited samples, as $t$ increases, $n$ decreases and $\mu$ increases. For the annealed ones, the trend is reversed, as $t$ increases, $n$ increases and $\mu$ decreases. While the effect of annealing led to an increase in $n$ and a decrease in $\mu$. Thus, the mobility values seem to be dictated by the carrier concentration ones. The origin of $n$ and $\mu$ dependences on the thickness may depend on several factors such as texture; it is found in the literature that $n$ and $\mu$ of ITO thin films may increase with thickness for one texture and decrease for another [28], i.e., a change in texture may have an effect on these parameters as reported by Agashe et al [30]. In the present work, we noted that annealing did affect the texture (the $\langle 100\rangle$ texture becomes stronger). This may have changed $n$ and $\mu$ dependences on thickness.

\section{Conclusion}

RF-sputtered ITO thin films on $\mathrm{Si}(100)$ substrates were investigated in the 61-768 nm thickness range. All films were found polycrystalline with the $\langle 100\rangle$ texture. The samples are subjected to a small growth-induced compressive stress, which decreases with increasing thickness $t$. There is an overall decrease of the grain size $D$ as $t$ increases, except for the $264 \mathrm{~nm}$ thick sample where a small increase in $D$ is observed. The surface appears smooth with pyramid-shaped grains and the roughness (rms) values increasing with $t$. The electrical resistivity $\rho$ increases with increasing $t$. This indicates that scattering at the surface may not be the major origin of the $\rho$ behaviour in this thickness range. As $t$ increases, the bulk carrier concentration $n$ decreases, while the mobility $\mu$ and the magnetoresistance $\Delta R$ increases. Annealing of ITO/Si films at $400^{\circ} \mathrm{C}$ in air improves the crystalline structure, the $\langle 100\rangle$ texture becomes stronger. After annealing, stress is relieved; the grain size and rms values increase. These structural changes did affect the electrical properties. Annealing led to a decrease in $\rho, \mu$ and the magnetoresistance, while $n$ increased. The decrease in $\rho$ might be attributed to the increase of grain size (scattering at the grain boundaries) and to the stronger texture; while the decrease in $\mu$ is probably due to the increase of $n$ rather than to the decrease of $\rho$. It is interesting to note that the variations of $n$ and $\mu$ with $t$ are different before and after annealing. For as-deposited samples, as $t$ increases, $n$ decreases and $\mu$ increases. For the annealed ones, the trend is reversed, as $t$ increases, $n$ increases and $\mu$ decreases.

\section{References}

[1] Hotovy J, Hupkes J, Bottler W, Marins E, Spiess L, Kups T et al 2013 Appl. Surf. Sci. 26981

[2] Manavizadeh N, Boroumand F A, Asl-Soleimani E, Raissi F, Bagherzadeh S, Khodayari A et al 2009 Thin Solid Films 517 2324

[3] Erjing Z, Weijia Z, Jun L, Dongjie Y, Jacques H J and Jing Z 2011 Vacuum 86290

[4] Betz U, Kharrazi Olsson M, Marthy J, Escolá M F and Atamny F 2006 Surf. Coat. Technol. 2005751

[5] Lee S-M, Lee Y-S, Shim C-H, Choi N-J, Joo B-S, Song K-D et al 2003 Sens. Actuat. B 9331

[6] Kim H, Piqué A, Horwitz J S, Mattoussi H, Murata H, Kafafi Z H et al 1999 Appl. Phys. Lett. 7423

[7] Lin H K and Hsu W C 2014 Appl. Surf. Sci. 3088

[8] Sathiaraj T S 2008 Microelectron. J. 391444

[9] Manavizadeh N, Khodayari A, Asl Soleimani E, Bagherzadeh S and Maleki M H 2009 Iranian J. Chem. Chem. Eng. (IJCCE) 2857

[10] Kerkache L, Layadi A, Dogheche E and Rémiens D 2007 Eur. Phys. J. Appl. Phys. 391

[11] Tuna O, Selamet Y, Aygun G and Ozyuzer L 2010 J. Phys. D: Appl. Phys. 43055402

[12] Song S, Yang T, Liu J, Xin Y, Li Y and S Han S 2011 Appl. Surf. Sci. 2577061

[13] Maurya D K 2007 Microelectron. J. 3876

[14] Lien S-Y 2010 Thin Solid Films 518 S10

[15] Socol G, Socol M, Stefan N, Axente E, Popescu-Pelin G, Craciun D et al 2012 Appl. Surf. Sci. 26042

[16] El Rhaleb H, Benamar E, Rami M, Roger J P, Hakam A and Ennaoui A 2002 Appl. Surf. Sci. 201138

[17] Venkatachalam S, Nanjo H, Kawasaki K, Wakui Y, Hayashi H and Ebina T 2011 Appl. Surf. Sci. 2578923

[18] Daoudi K, Canut B, Blanchin M G, Sandu C S, Teodorescu V S and Roger J A 2002 Mater. Sci. Eng. C 21313

[19] Kerkache L, Layadi A and Mosser A 2009 J. Alloys Compd. 48546

[20] Kerkache L, Layadi A, Doghache H and Riemens R $2006 J$. Phys. D: Appl. Phys. 39184

[21] Rao A V, Mansour S A and Bement A L 1996 J. Mater. Lett. 29255

[22] Kerkache L, Layadi A, Dogheche H and Riemens R $2011 \mathrm{~J}$. Alloys Compd. $\mathbf{5 0 9} 6072$

[23] Sandoval-Paz M G and Ramirez-Bon R 2009 Thin Solid Films 5172596

[24] Eberhart J P 1989 Analyse structural et chimique des matériaux. Bordas (Paris)

[25] Kim H and Gilmore C M 1999 J. Appl. Phys. 866451

[26] Balasundaraprabhu R, Monakhov E V, Muthukumarasamy N, Nilsen O and Svenss B G 2009 Mater. Chem. Phys. 114425

[27] Shen L, Ren Y, Yang D, Xing Y, Sun G, Liu J et al 2015 J. Alloys Compd. 632218

[28] Guittoum A, Kerkache L and Layadi A 1999 Eur. Phys. J. Appl. Phys. 7201

[29] Zheng H, Li L, Sun Z, Yu S and Luo W 2016 Appl. Surf. Sci. 362230

[30] Agashe C and Mahamuni S 1995 Semicond. Sci. Technol. 10 172 